\title{
Short communication: Intra- and inter-individual milk microbiota variability in healthy and infected water buffalo udder quarters
}

\author{
Carlotta Catozzi, ${ }^{1 *}$ Anna Cuscó, ${ }^{2}$ Cristina Lecchi, ${ }^{1}$ Andrea Talenti, ${ }^{3}$ Alessandra Martucciello, ${ }^{4}$ \\ Giovanna Cappelli, ${ }^{4}$ Armand Sanchez Bonastre, ${ }^{5}$ Olga Francino, ${ }^{5}$ and Fabrizio Ceciliani ${ }^{1}$ \\ ${ }^{5}$ Molecular Genetics Veterinary Service, Veterinary School, Universitat Autònoma de Barcelona, 08193 Bellaterra, Barcelona, Spain
}

\section{ABSTRACT}

The concept that ruminant mammary gland quarters are anatomically and physiologically unrelated has been recently challenged by immunological evidence. How this interdependence reflects on individual quarter milk microbiota is unknown. The aim of the present study was to cover this gap by investigating the interdependence of quarters among the same mammary gland at the milk microbiota level using next-generation sequencing of the V4-16S rRNA gene. A total of 52 samples were included in this study and classified as healthy or affected by subclinical mastitis. Extraction of DNA, amplification of the V4-16S rRNA gene, and sequencing using Ion Torrent Personal Genome Machine (Thermo Fisher Scientific, Waltham, MA) were carried out. We found that the intra-individual variability was lower than the inter-individual one. The present findings further support at milk microbiota level the hypothesis of the interdependence of quarters, as previously demonstrated following immunological studies, suggesting that individual factors (e.g., immunity, genetics) may have a role in modulating milk microbiota.

Key words: quarter interdependence, water buffalo, milk microbiota

\section{Short Communication}

Mammary gland quarters within dairy cows have been regarded as independent of each other, given the background that each quarter has its own vascular system, nerve supply, and suspensory apparatus (Berry and Meaney, 2006; Akers and Nickerson, 2011). Preliminary investigations on immune-related cells

Received January 22, 2019.

Accepted April 16, 2019.

*Corresponding author: carlotta.catozzi@unimi.it suggesting that mammary gland quarters do not act independently during mastitis (Merle et al., 2007) were further confirmed by the evidence that the infection of one udder quarter also influences other uninfected quarters (Mitterhuemer et al., 2010; Jensen et al., 2013). More recent studies demonstrated that quarters of infected udders influence the percentage of B cells and the expression of adhesion molecules in neutrophils of uninfected quarters (Blagitz et al., 2015).

To the best of our knowledge, the difference in bacterial taxonomy between quarters within the same udder has not been investigated yet, except in human breast milk, where high intra-individual similarity between individuals was demonstrated (Avershina et al., 2018). Culture-independent methodologies relying on highthroughput DNA sequencing of $16 \mathrm{~S}$ (next-generation sequencing) are currently applied to describe the relationship between resident microbial population and the development of mastitis and allow for an in-depth description of species that cannot be cultured (Oikonomou et al., 2012, 2014; Lima et al., 2018), and are regarded as the ideal techniques to identify differences between quarter milk microbiota.

The domestic water buffalo (Bubalus bubalis) provides a significant amount of global milk production and is the major milk-producing animal in several countries, such as India and Pakistan (FAO, 2016). Water buffalo udder quarters are regarded as anatomically and physiologically independent of the others within the same mammary gland, as in the cow (Thomas et al., 2004; Ambord et al., 2010). How this anatomical independence is related to immunological and microbiological status is unknown. Starting from previous results about water buffalo milk microbiota (Catozzi et al., 2017), this study aimed to elucidate the interdependence of quarters by investigating the variability of milk microbiota in composition and structure between healthy quarters within the same udder. To assess whether modification 
in unhealthy status, such as mild inflammation, reflects on the other quarters, the composition of microbiota in milk from animals affected by subclinical mastitis was also determined.

Water buffalo quarter milk samples were collected from healthy and subclinical mastitis-affected quarters. Sixteen animals were enrolled from the same farm to reduce the microbiota variability due to different management and feeding regimen, and were homogeneous for parity (from second to fourth milking) and stage of lactation (mid lactation). A total of 52 milk quarter samples, of which 18 healthy (from 6 animals) and 34 affected by subclinical mastitis (from 10 animals), were collected. Healthy quarters were characterized by absence of clinical symptoms, negative microbiological culture for mastitis pathogens, and a SCC lower than 200,000 cells/mL; subclinical mastitis samples were defined by absence of clinical symptoms, positive microbiological culture for mastitis pathogens, SCC higher than 200,000 cells/mL, or both. The number of the included quarters is from 2 to 4 per animal; the list of animals enrolled and the details of their clinical status are presented in Supplemental Table S1 (https://doi .org/10.3168/jds.2019-16352).

Mammary glands were disinfected, the first 3 strains of milk were discarded, and gloves were changed after every milk collection to prevent any contamination. Milk samples were collected, immediately refrigerated, and delivered to the laboratory for microbiological and SCC analysis.

Microbiological culture tests and SCC were performed as previously reported (Catozzi et al., 2017). The DNA extraction was carried out as previously reported as well (Catozzi et al., 2017). Briefly, $1 \mathrm{~mL}$ of milk was centrifuged at room temperature at $16,100 \times g$ for $20 \mathrm{~min}$. Fat and supernatant were removed and the remaining pellet was resuspended with $250 \mu \mathrm{L}$ of the Power Bead Tube of the DNeasy Power Soil Kit (Qiagen, Hilden, Germany) used to extract bacterial DNA, according to the manufacturer's instructions. The V4 region of $16 \mathrm{~S}$ rRNA gene was amplified for each sample. The forward primer was 5'-CCATCTCATCCCTGCGTGTCTCCGACTCAG $N N N N N N N N N N N N N N N N N G$ ATGTGYCAGCMGCCGCGGTAA-3', and was composed of the adapter linker, the key, the sample-specific barcode, and the $515 \mathrm{~F}$ forward primer. The reverse primer was 5'-CTCTCTATGGGCAGTCGGTGATGGACTACNVGGGTWTCTAAT-3', composed of the adapter linker and the R806 reverse primer. The Thermo Scientific Phusion Hot Start II High-Fidelity DNA polymerase kit (Thermo Fisher Scientific, Waltham, MA) was used to perform V4 PCR (Catozzi et al., 2017). Next-generation sequencing was carried out using an Ion Torrent Personal Genome Machine with the Ion
318 Chip Kit v2 (Thermo Fisher Scientific), following the manufacturer's instructions. Raw sequences have been submitted to the National Center for Biotechnology Information under Bioproject accession number PRJNA492401. Reads were demultiplexed and analyzed using Quantitative Insight Into Microbial Ecology 2 software (QIIME 2; https://qiime2.org; Caporaso et al., 2011). Briefly, DADA2 was used as quality filtering method to denoise, dereplicate single-end sequences, and remove chimeras (Callahan et al., 2016); a truncation length of 245 bases was used. After that, the units of observation, composed of unique sequences, namely amplicon sequence variants (ASV), were used to classify and assign taxonomy by Greengenes 13.8 (DeSantis et al., 2006) at $99 \%$ of operational taxonomic unit identity and trimmed to V4 region as reference database. The filtered feature table was used to perform the downstream analysis. The raw and final count of sample sequences, together with the number of ASV, is included in Supplemental File S1 (https://doi.org/ 10.3168/jds.2019-16352). Taxonomic analysis was performed for each sample or sample group at the phylum and family level with a relative abundance of at least $1 \%$. Results and taxonomic classification are presented in Figure 1 at phylum (panel A) and family (panel B) level and Supplemental Table S2 (https://doi.org/ 10.3168/jds.2019-16352). Detailed results are included in Supplemental File S1 (https://doi.org/10.3168/jds .2019-16352). It was found that Actinobacteria, Bacteroidetes, Firmicutes, and Proteobacteria predominate in the milk microbiota at the phylum level (Supplemental Table S2, panel A, https://doi.org/10.3168/jds.2019 -16352), whereas Aerococcaceae, Corynebacteriaceae, Moraxellaceae, Staphylococcaceae, and Propionibacteriaceae provided the most abundant taxa at the family level (Supplemental Table S2, panel B, https://doi.org/ 10.3168/jds.2019-16352).

The number of studies carried out in milk water buffalo is too limited to identify a common healthy and unhealthy microbiota. In bovine milk, beside those related to inflammation (Oikonomou et al., 2014; Catozzi et al., 2017; Lima et al., 2017), variations in milk microbiota have been linked not only to antibiotic treatment (Ganda et al., 2016, 2017), as expected, but also to lactation stage, weather conditions, and diet supplementation (Chaves Lopez et al., 2016; Li et al., 2018), suggesting the presence of a wide range of factors and sources influencing the milk microbial community (Derakhshani et al., 2018). Therefore, possible variations of the relative abundance at phyla and family levels found in this study as compared with previous ones could be related to different management conditions.

Beta diversity, which is a measure of the differences occurring between samples by estimating how many 
A

- Firmicutes

- Proteobacteria

- Actinobacteria

- Bacteroidetes

- Other

None

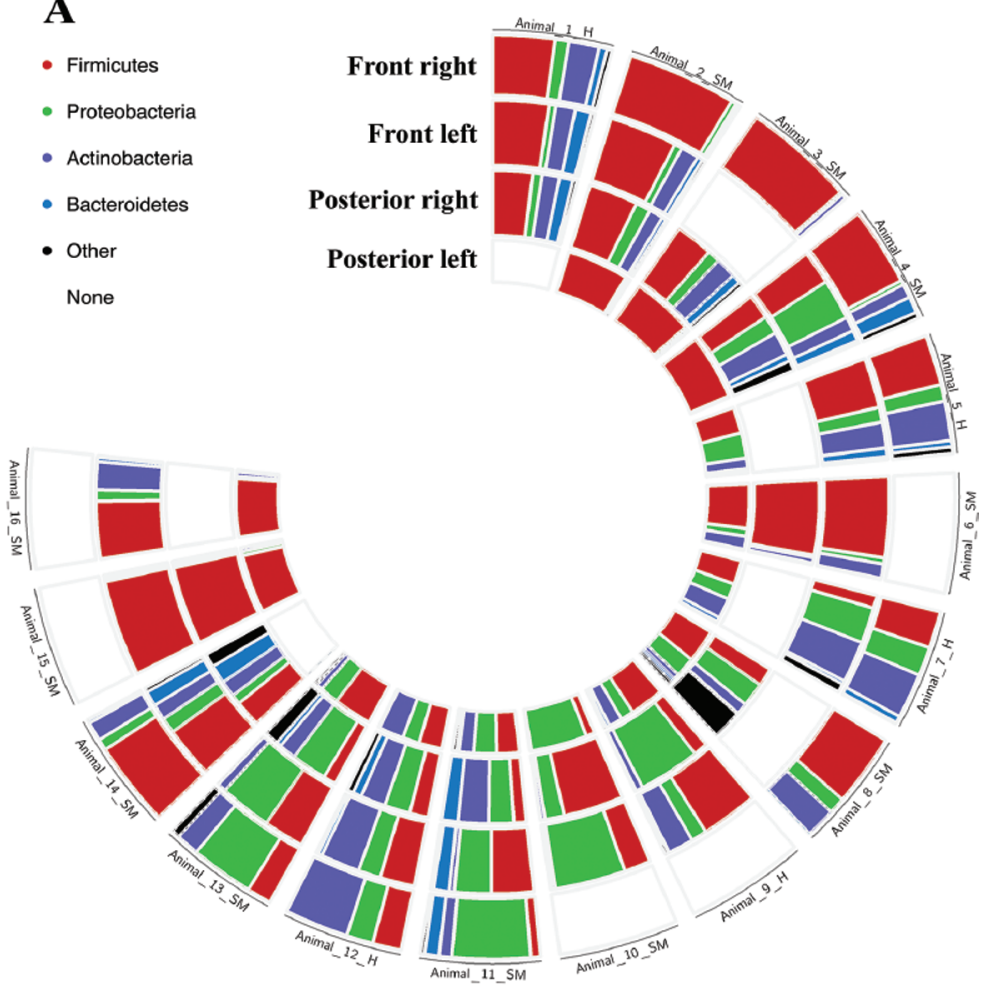

B

- Moraxellaceae
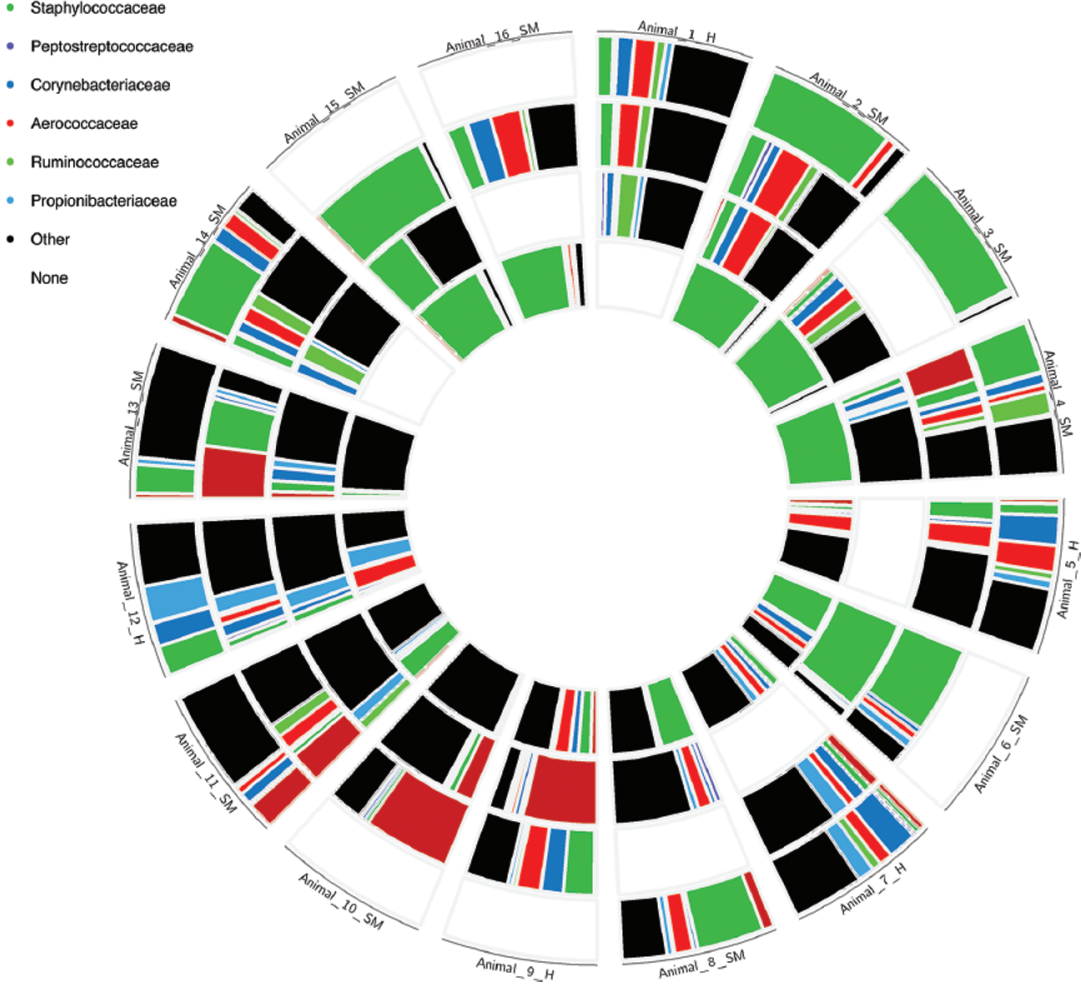

Figure 1. Taxonomic results at phylum (panel A, relative average abundance $\geq 1 \%$ ) and family level (panel B, relative average abundance $\geq 2.5 \%$ ) for all animal quarters. Each slice corresponds to one animal and each circle section corresponds to a quarter. The microbiological culture result for mastitis pathogens is indicated below each quarter. From the outer to the inner part: front right, front left, posterior right, posterior left quarters. White quarters indicate that the sample is missing. $\mathrm{H}=$ healthy; $\mathrm{SM}=$ subclinical mastitis. 
taxa they share, was performed using qualitative and quantitative approaches and taking into account the taxa phylogeny (unweighted and weighted UniFrac distances matrices, respectively). Bray-Curtis dissimilarity analysis, which does not rely on taxa genetic relatedness, was also assessed. Diversity analysis was assessed using a depth of 17,500 sequences per sample. Abundances for phyla and families were represented using the CIRCOS software (http://circos.ca/software/ ; Krzywinski et al., 2009). Wilcoxon signed pairwise test was performed for unpaired comparisons among $\beta$ diversity matrices from quarters within the same animal and between different animals using pairwise. wilcox.test function in R (http://www.R-project.org). After false discovery rate correction, comparisons were considered statistically significant at $P<0.05$. The detailed workflow used in QIIME and in $\mathrm{R}$ is shown in Supplemental File S2 (https://doi.org/10.3168/jds .2019-16352).

We obtained a mean of $10.33( \pm 7.7)$ and $15.3( \pm 29.5)$ ASV shared within the same individuals for healthy and subclinical mastitis-affected quarters, respectively. The range of shared ASV between subclinical mastitisaffected quarters of the same animal was from 2 to 10, with one exception in one animal (with 99 shared ASV) that can explain the great variability observed for subclinical mastitis-affected status. In fact, excluding this individual, the shared ASV mean decreases to $6( \pm 2.8)$. Conversely, healthy quarters within the same mammary gland exhibit a higher range of shared ASV (from 4 to 24). One ASV, belonging to the Propionibacteriaceae family, was detected in all samples from this study, followed by Staphylococcaceae present in 83 and $88 \%$ of healthy and subclinical mastitis-affected quarters, respectively. Results of shared ASV are presented in Supplemental File S1 (https://doi.org/10.3168/jds .2019-16352).

The individual variation in the amount of the most abundant phyla (panel A) and families (panel B) is reported in Figure 2 and Supplemental Table S3 (https:/ /doi.org/10.3168/jds.2019-16352). The violin plot indicates the range of standard deviations (SD) of the main taxa, through which it is possible to evaluate the range of intra-individual variability for all animals; the SD of each taxon relative abundance, plotted in the y-axis of Figure 2, represents the mean of the SD of healthy and subclinical mastitis-affected animals, indicating how much each taxon varies within individuals. The closer the $\mathrm{SD}$ value is to 0 , the smaller is the variation.

Proteobacteria and Firmicutes showed the highest variation in healthy (SD of 15 and 13\%, respectively) and subclinical mastitis-affected samples (SD of 22 and $28 \%$, respectively; Supplemental Table S2, panel A, https://doi.org/10.3168/jds.2019-16352). At the indi- vidual level, Firmicutes represented the most variable phylum in the subclinical mastitis-affected samples (SD mean of $19 \%$ ranging from 3.1 to $30.6 \%$ ), as compared with the healthy ones (SD mean of $9 \%$ ranging from 2.6 to $21.2 \%$ ), followed by Proteobacteria (SD mean of 12 and $9 \%$ for healthy and subclinical mastitis-affected samples, respectively; Supplemental Table S3, panel A, https://doi.org/10.3168/jds.2019-16352). This result may potentially explain the differences found in microbiota from previous report (Catozzi et al., 2017). The other main phyla, namely Acidobacteria and Bacteroidetes, were more stable, reaching a SD mean lower than $8 \%$ in healthy quarters (Supplemental Table S3, panel A, https://doi.org/10.3168/jds.2019-16352). Staphylococcaceae and Moraxellaceae were the most variable families for subclinical mastitis-affected and healthy samples with a SD of 34 and $16 \%$, respectively (Supplemental Table S2, panel B, https://doi.org/10 $.3168 /$ jds.2019-16352). At the individual level, these families showed the greatest variability in healthy samples (Moraxellaceae with a SD mean of $10 \%$ ranging from 0.9 to $37 \%$ ) and subclinical mastitis-affected samples (Staphylococcaceae with a SD mean of $28 \%$ ranging from 2.5 to $49 \%$; Supplemental Table S3, panel B, https://doi.org/10.3168/jds.2019-16352), whereas Propionibacteriaceae, Corynebacteriaceae, and Aerococcaceae were the most stable. We found that the most stable phylum across healthy or subclinical mastitisaffected quarters was represented by Bacteroidetes. At family level, the relative abundance of Propionibacteriaceae showed the greatest stability, followed by $\mathrm{Co}$ rynebacteriaceae and Aerococcaceae. On the contrary, Firmicutes and Proteobacteria were the most variable phyla in both healthy and subclinical mastitis-affected quarter milk samples; as expected, the families Staphylococcaceae and Moraxellaceae showed the greatest variation in relative abundance. At the family level, the mean and median variability within animals was always lower than 6\%, with the exception of Staphylococcaceae and Moraxellaceae.

The comparison between quarter milk microbiota within the same individual and between different individuals was performed using unweighted, weighted UniFrac, and Bray-Curtis distance matrices. Results are presented in Supplemental Table S4, https://doi.org/10 $.3168 /$ jds.2019-16352; values close to 0 are representative of high similarity, whereas values close to 1 show a lower similarity. A box plot with statistical significant differences is presented in Figure 3. Healthy and subclinical mastitis-affected quarters within individuals showed more similarity in terms of microbiota structure as compared with those between individuals by using Unifrac analysis. Bray-Curtis dissimilarity also confirmed the presence of a higher similarity within quar- 
ters in healthy individuals. The dissimilarity between quarter samples within and between individuals was also plotted by using nonmetric multidimensional scaling for healthy and subclinical mastitis-affected quarters (Supplemental Figure S1; https://doi.org/10.3168/ jds.2019-16352). Previous studies have demonstrated the communication among quarters at the immunological level (Burvenich et al., 2003; Merle et al., 2007; Jensen et al., 2013; Blagitz et al., 2015). We presented the evidence that, in water buffaloes as well, concerning the milk microbiota structure, the intra-individual variability was lower than the inter-individual variability in both healthy and subclinical mastitis-affected quarters. The present finding is partially consistent with what has been recently reported in human milk (Avershina et al., 2018), which demonstrated a high intra-individual similarity between microbiota of milk collected from 2 mammary glands. In fact, we found that, in healthy samples, the similarity was greater in quarters within the same udder rather that between different mammary glands, as demonstrated by UniFrac and Bray-Curtis diversity analysis. The same profile was also demonstrated also in subclinical mastitis groups by means of the weighted UniFrac analysis.

Subclinical mastitis quarters showed a greater dissimilarity compared with the healthy ones, consistent with previous studies in water buffaloes and cows (Oikonomou et al., 2014; Catozzi et al., 2017), demonstrating that the development of a disease destabilizes the microbiota rather than shifting to a determined structure (Zaneveld et al., 2017).

The new concept of hologenome, defined as hostmicrobe genomes as a unit of evolution, is taking shape (Zilber-Rosenberg and Rosenberg, 2008; Shapira, 2016), meaning that selection processes involve the genomes of both the individual and microorganisms. Here, we support the presence of the quarter's interdependence at the milk microbiota level, showing that the intra-in-

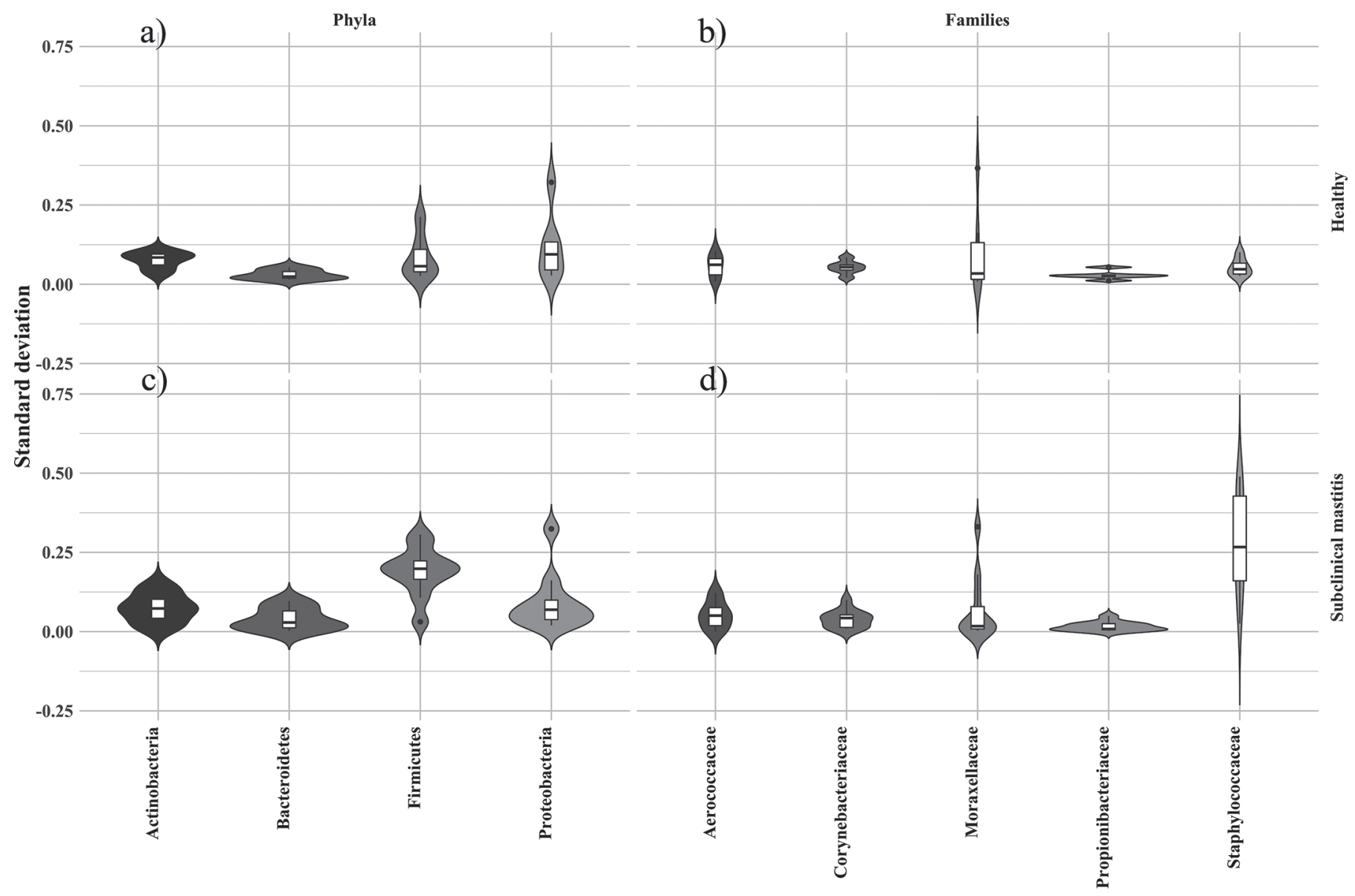

Figure 2. Violin plots of the SD for each individual for the main phyla (a and c) and families (b and d). The relative average abundance was $\geq 1 \%$. Violin plots for (a) phyla and (b) family variability in healthy animals, as well as (c) phyla and (d) family variability in subclinical mastitis-affected animals were created from the mean of the single SD for each animal for each taxon. The violin plot is composed of a white box with the median (line within the box), upper and lower quartiles (ends of the box), and highest and lowest value (extreme lines). The different width around the white box indicates the distribution shape of the data. 


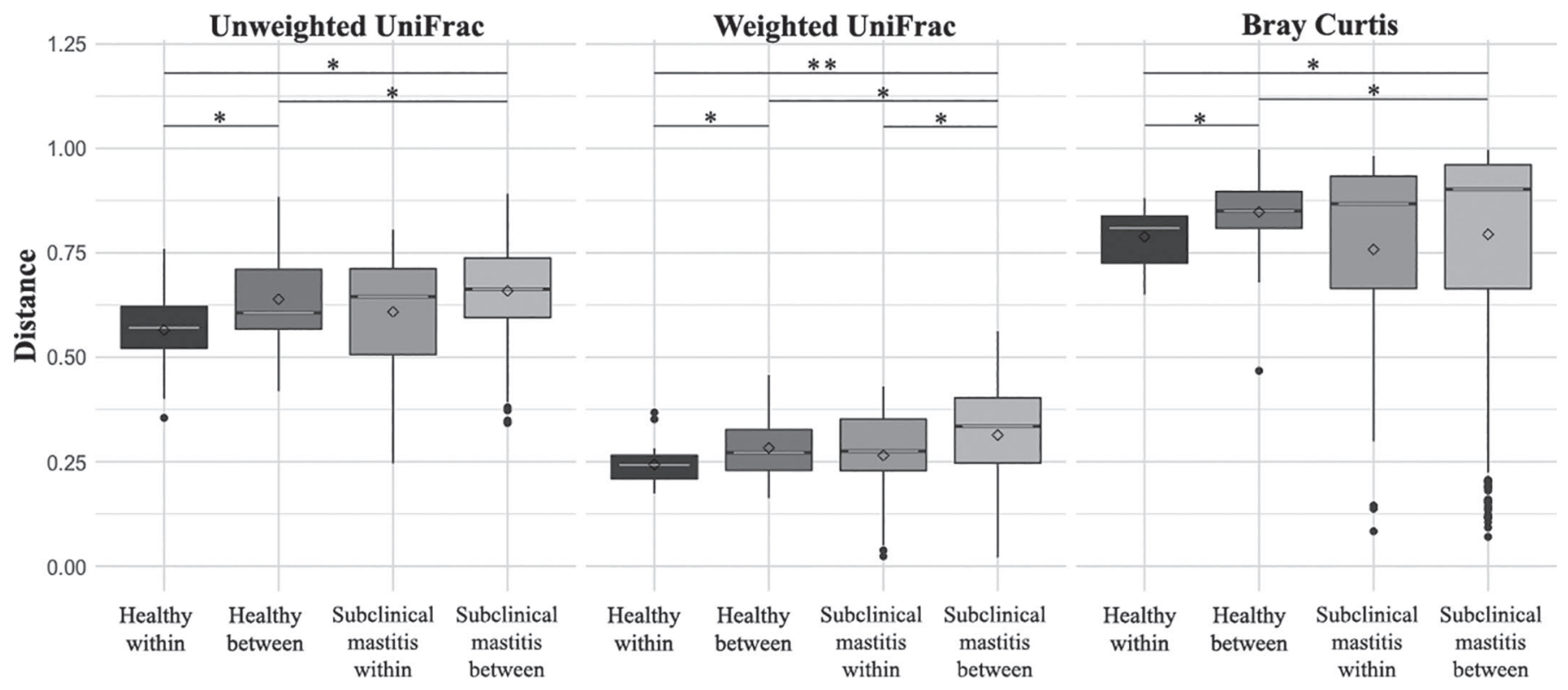

Figure 3. Box plots of unweighted and weighted UniFrac distance matrices and Bray-Curtis dissimilarity. Median (line within the box), mean (diamond shape), upper and lower quartiles (ends of the box), and highest and lowest value (extreme lines) are shown. Outiers are indicated by black points. Statistical significance is presented where $0.05<P<0.001\left(^{*}\right)$ and $P<0.001(* *)$. False discovery rate correction was applied.

dividual similarity was greater than the inter-individual one.

In conclusion, the results provided in this preliminary study demonstrated that the microbiota of the 4 quarters of the water buffalo udder cannot be regarded as separate entities. Further investigation is required to confirm the present results in bovine species.

\section{ACKNOWLEDGMENTS}

This work was supported by Progetti di Recerca Corrente, AM grant no. IZS ME 10/13 RC.

\section{REFERENCES}

Akers, R. M., and S. C. Nickerson. 2011. Mastitis and its impact on structure and function in the ruminant mammary gland. J. Mammary Gland Biol. Neoplasia 16:275-289. https://doi.org/10.1007/ s10911-011-9231-3.

Ambord, S., M. H. Stoffel, and R. M. Bruckmaier. 2010. Teat anatomy affects requirements for udder preparation in Mediterranean buffaloes. J. Dairy Res. 77:468-473. https://doi.org/10.1017/ S0022029910000518.

Avershina, E., I. L. Angell, M. Simpson, O. Storrø, T. Øien, R. Johnsen, and K. Rudi. 2018. Low maternal microbiota sharing across gut, breast milk and vagina, as revealed by $16 \mathrm{~s}$ rRNA gene and reduced metagenomic sequencing. Genes (Basel) 9. https://doi.org/ $10.3390 /$ genes 9050231

Berry, D. P., and W. J. Meaney. 2006. Interdependence and distribution of subclinical mastitis and intramammary infection among udder quarters in dairy cattle. Prev. Vet. Med. 75:81-91. https:// doi.org/10.1016/j.prevetmed.2006.02.001.

Blagitz, M. G., F. N. Souza, C. F. Batista, S. A. Diniz, L. F. F. Azevedo, M. X. Silva, J. P. A. Haddad, M. B. Heinemann, M. M. O. P. Cerqueira, and A. M. M. P. Della Libera. 2015. Flow cytometric analysis: Interdependence of healthy and infected udder quarters. J. Dairy Sci. 98:2401-2408. https://doi.org/10.3168/jds.2014-8727.

Burvenich, C., V. Van Merris, J. Mehrzad, A. Diez-Fraile, and L. Duchateau. 2003. Severity of E. coli mastitis is mainly determined by cow factors. Vet. Res. 34:521-564. https://doi.org/10.1051/vetres: 2003023.

Callahan, B. J., P. J. Mcmurdie, M. J. Rosen, A. W. Han, A. J. Johnson, and S. P. Holmes. 2016. DADA2: High resolution sample inference from Illumina amplicon data. Nat. Methods 13:581-583. https://doi.org/10.1038/nmeth.3869.

Caporaso, J. G., J. Kuczynski, J. Stombaugh, K. Bittinger, F. D. Bushman, E. K. Costello, N. Fierer, A. G. Peña, K. Goodrich, J. I. Gordon, G. A. Huttley, S. T. Kelley, D. Knights, J. E. Koenig, R. E. Ley, C. A. Lozupone, D. McDonald, B. D. Muegge, M. Pirrung, J. Reeder, J. R. Sevinsky, P. J. Turnbaugh, W. A. Walters, J. Widmann, T. Yatsunenko, J. Zaneveld, and R. Knight. 2011. QIIME allows analysis of high-throughput community sequencing data. Nat. Methods 7:335-336. https://doi.org/10.1038/nmeth.f.303.

Catozzi, C., A. Sanchez Bonastre, O. Francino, C. Lecchi, E. De Carlo, D. Vecchio, A. Martucciello, P. Fraulo, V. Bronzo, A. Cuscó, S. D'Andreano, and F. Ceciliani. 2017. The microbiota of water buffalo milk during mastitis. PLoS One 12:e0184710. https://doi.org/ 10.1371/journal.pone.0184710.

Chaves Lopez, C., A. Serio, C. Rossi, G. Mazzarrino, S. Marchetti, F. Castellani, L. Grotta, F. P. Fiorentino, A. Paparella, and G. Martino. 2016. Effect of diet supplementation with Ascophyllum nodosum on cow milk composition and microbiota. J. Dairy Sci. 99:6285-6297. https://doi.org/10.3168/jds.2015-10837.

Derakhshani, H., K. B. Fehr, S. Sepehri, D. Francoz, J. De Buck, H. W. Barkema, J. C. Plaizier, and E. Khafipour. 2018. Invited review: Microbiota of the bovine udder: Contributing factors and potential implications for udder health and mastitis susceptibility. J. Dairy Sci. 101:10605-10625. https://doi.org/10.3168/jds.2018 -14860 .

DeSantis, T. Z., P. Hugenholtz, N. Larsen, M. Rojas, E. L. Brodie, K. Keller, T. Huber, D. Dalevi, P. Hu, and G. L. Andersen. 2006. Greengenes, a chimera-checked $16 \mathrm{~S}$ rRNA gene database and workbench compatible with ARB. Appl. Environ. Microbiol. 72:5069-5072. https://doi.org/10.1128/AEM.03006-05. 
FAO. 2016. FAO report. Accessed Jan. 10, 2016. http://www.fao.org/ agriculture/dairy-gateway/milk-production/dairy-animals/water -buffaloes/en/\#.V-8-cREfK8.

Ganda, E. K., R. S. Bisinotto, S. F. Lima, K. Kronauer, D. H. Decter, G. Oikonomou, Y. H. Schukken, and R. C. Bicalho. 2016. Longitudinal metagenomic profiling of bovine milk to assess the impact of intramammary treatment using a third-generation cephalosporin. Sci. Rep. 6:37565. https://doi.org/10.1038/srep37565.

Ganda, E. K., N. Gaeta, A. Sipka, B. Pomeroy, G. Oikonomou, Y. H Schukken, and R. C. Bicalho. 2017. Normal milk microbiome is reestablished following experimental infection with Escherichia coli independent of intramammary antibiotic treatment with a thirdgeneration cephalosporin in bovines. Microbiome 5:74. https://doi .org/10.1186/s40168-017-0291-5.

Jensen, K., J. Günther, R. Talbot, W. Petzl, H. Zerbe, H. J. Schuberth, H. M. Seyfert, and E. J. Glass. 2013. Escherichia coli- and Staphylococcus aureus-induced mastitis differentially modulate transcriptional responses in neighbouring uninfected bovine mammary gland quarters. BMC Genomics 14. https://doi.org/10.1186/1471 -2164-14-36.

Krzywinski, M., J. Schein, I. Birol, R. Gascoyne, D. Horsman, S. J. Jones, and M. A. Marra. 2009. Circos: An information aesthetic for comparative genomics. Genome Res. 19:1639-1645. https://doi .org/10.1101/gr.092759.109.

Li, N., Y. Wang, C. You, J. Ren, W. Chen, H. Zheng, and Z. Liu. 2018. Variation in raw milk microbiota throughout 12 months and the impact of weather conditions. Sci. Rep. 8:2371. https://doi.org/10 .1038/s41598-018-20862-8.

Lima, S. F., M. L. S. Bicalho, and R. C. Bicalho. 2018. Evaluation of milk sample fractions for characterization of milk microbiota from healthy and clinical mastitis cows. PLoS One 13:e0193671. https: /doi.org/10.1371/journal.pone.0193671.

Lima, S. F., A. G. V. Teixeira, F. S. Lima, E. K. Ganda, C. H. Higgins, G. Oikonomou, and R. C. Bicalho. 2017. The bovine colostrum microbiome and its association with clinical mastitis. J. Dairy Sci. 100:3031-3042. https://doi.org/10.3168/jds.2016-11604.

Merle, R., A. Schröder, and J. Hamann. 2007. Cell function in the bovine mammary gland: A preliminary study on interdependence of healthy and infected udder quarters. J. Dairy Res. 74:174-179. https://doi.org/10.1017/S002202990600238X.

Mitterhuemer, S., W. Petzl, S. Krebs, D. Mehne, A. Klanner, E. Wolf, H. Zerbe, H. Blum, and M. K. Siddiq. 2010. Escherichia coli infection induces distinct local and systemic transcriptome responses in the mammary gland. BMC Genomics 11:138.

Oikonomou, G., M. L. Bicalho, E. Meira, R. E. Rossi, C. Foditsch, V. S. Machado, A. G. V. Teixeira, C. Santisteban, Y. H. Schukken, and R. C. Bicalho. 2014. Microbiota of cow's milk; distinguishing healthy, sub-clinically and clinically diseased quarters. PLoS One 9:e85904. https://doi.org/10.1371/journal.pone.0085904.

Oikonomou, G., V. S. Machado, C. Santisteban, Y. H. Schukken, and R. C. Bicalho. 2012. Microbial diversity of bovine mastitic milk as described by pyrosequencing of metagenomic 16 s rDNA. PLoS One 7. https://doi.org/10.1371/journal.pone.0047671.

Shapira, M. 2016. Gut microbiotas and host evolution: Scaling up symbiosis. Trends Ecol. Evol. 31:539-549. https://doi.org/10.1016/j .tree.2016.03.006.

Thomas, C. S., K. Svennersten-Sjaunja, M. R. Bhosrekar, and R. M. Bruckmaier. 2004. Mammary cisternal size, cisternal milk and milk ejection in Murrah buffaloes. J. Dairy Res. 71:162-168.

Zaneveld, J. R., R. McMinds, and R. V. Thurber. 2017. Stress and stability: Applying the Anna Karenina principle to animal microbiomes. Nat. Microbiol. 2:17121. https://doi.org/10.1038/nmicrobiol .2017 .121 .

Zilber-Rosenberg, I., and E. Rosenberg. 2008. Role of microorganisms in the evolution of animals and plants: The hologenome theory of evolution. FEMS Microbiol. Rev. 32:723-735. https://doi.org/10 .1111/j.1574-6976.2008.00123.x. 